\title{
Treatment outcome of gartland type III supracondylar fracture humerus in children with closed reduction and percutaneous pinning
}

\author{
P. Thomas George*, Mithun Joy Kattoor, Samson Samuel Edayalamuriyil
}

Department of Orthopaedics, Pushpagiri Medical College, Thiruvalla, Kerala, India

Received: 22 July 2016

Accepted: 26 July 2016

\section{*Correspondence:}

Dr. P. Thomas George,

E-mail: thomas_theone86@yahoo.co.in

Copyright: (c) the author(s), publisher and licensee Medip Academy. This is an open-access article distributed under the terms of the Creative Commons Attribution Non-Commercial License, which permits unrestricted non-commercial use, distribution, and reproduction in any medium, provided the original work is properly cited.

\begin{abstract}
Background: Treatment of Gartland's type III supracondylar fracture of humerus in children is one of the most challenging one. Conservative management usually results in deformity. On the other hand open reduction and internal fixation is a more invasive surgical method with a long recovery period. Thus, this study presents cases treated by closed reduction and percutaneous pinning and discussed its anatomical results, functional results and lastly its complications.

Methods: Twenty five cases were treated with closed reduction and percutaneous pinning at Pushpagiri Medical College.

Results: Out of the 25 patients studied, 24 (96\%) had satisfactory results according to Modified Flynn's criteria and there was only one case of superficial pin tract infection with no cases of cubitus varus deformity.

Conclusions: This study presents that closed reduction and percutaneous pinning is a very good modality of treatment of Gartland type III fractures of humerus in children with very few complications.
\end{abstract}

Keywords: Closed reduction, Supracondylar fracture, Children, Gartland and Modified Flynn's criteria

\section{INTRODUCTION}

Supracondylar fracture of humerus is a common injury in children accounting $60 \%$ of the fractures around the elbow in children. ${ }^{1}$ The rate of occurrence increases steadily in the first five years of life to peak at 5-7 years of age. ${ }^{2}$

The management of displaced supracondylar fracture of the humerus is one of the most difficult of the many fractures seen in children. ${ }^{3}$ If the fracture is not treated properly it may give rise to many complications like Volkmann's ischemic contracture, neurovascular injury, skin slough, myositis ossificans, elbow stiffness and malunion. There is no controversy about management of the undisplaced fractures. But many methods have been proposed for the treatment of displaced supracondylar fractures of the humerus in children, such as closed reduction and plaster of Paris slab application, skin traction, overhead skeletal traction, open reduction and internal fixation, and closed reduction and percutaneous pin fixation. ${ }^{4}$

Closed reduction with plaster of Paris slab immobilization has traditionally been recommended for displaced supracondylar fractures, but loss of reduction and necessity of repeated manipulation is likely to go for malunion producing varus or valgus deformity of elbow and elbow stiffness. ${ }^{5}$ Traction (skin or skeletal), which has also been used for many years, has been shown to be safe and reliable, but it has the drawback of requiring a long stay in the hospital. ${ }^{4}$ 
Open reduction and internal fixation has generally been reserved for specific indications mainly for an open fracture, a fracture requiring vascular exploration, or an irreducible fracture. ${ }^{4}$

Thus, in our hospital we prefer closed reduction and percutaneous pinning for displaced fractures. Purpose of our study was to evaluate the age, sex and side incidence associated with these types of fractures and then anatomical and functional results and lastly complications in our patients treated with the above procedure.

\section{METHODS}

Twenty five patients with acute closed Gartland's type III fractures in patients with age less than 15 years were taken and patients whose age was above 15 years, open fractures, old fractures or associated nerve or vessel injuries were excluded.

Selected patients were admitted in our hospital and treated according to a standard protocol. Firstly AP and lateral view radiographs of the elbow joint were taken.

Classification system proposed by Gartland's was used which defines type-I fractures as undisplaced, type-II fractures as displaced fractures with intact posterior cortex and type III as displaced with no cortical contact and further subdivided it into posteromedial and posterolateral.

Thus Patients with type III fractures qualifying the inclusion criteria were accepted for the study and surgically treated within 48 hours of initial injury.

Table 1: Flynn's grading system.

\begin{tabular}{|llll|}
\hline Result & Rating & $\begin{array}{l}\text { Cosmetic } \\
\text { factor: } \\
\text { Carrying } \\
\text { angle loss } \\
\text { (degrees) }\end{array}$ & $\begin{array}{l}\text { Functional } \\
\text { factor: } \\
\text { motion } \\
\text { loss } \\
\text { (degrees) }\end{array}$ \\
\hline \multirow{3}{*}{ Satisfactory } & Excellent & $0-5$ & $0-5$ \\
\cline { 2 - 4 } & Good & $6-10$ & $6-10$ \\
\cline { 2 - 4 } & Fair & $11-15$ & $11-15$ \\
\hline Unsatisfactory & Poor & $>15$ & $>15$
\end{tabular}

Closed reduction and percutaneous pinning with two smooth $\mathrm{K}$-wires was performed under $\mathrm{C}$-arm guidance (Figure 1, 2 and 3). After the pin placement, elbow was extended and the carrying angle was measured and checked with the contra lateral side. Post operatively, the affected upper limb was immobilized in long arm cast for 4 weeks .Four weeks later after radiological evidence of fracture healing was observed on X-rays, the cast and $\mathrm{K}$ wires were removed. Active range of movements of the elbow was started. Further follow ups were done at 12 weeks and 24 weeks. The final results were evaluated using Modified Flynn's criteria. ${ }^{6}$ The results were graded as excellent, good, fair (satisfactory) and poor (unsatisfactory) according to motion loss and carrying angle loss (Table 1).
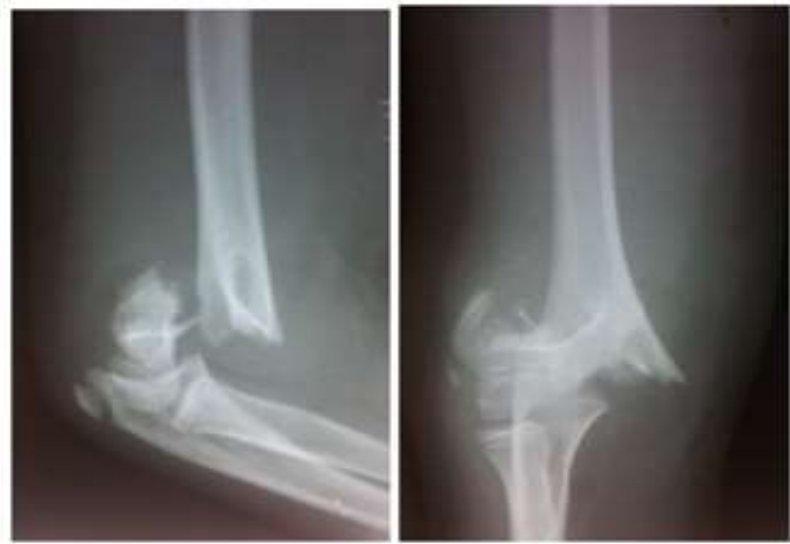

Figure 1: Anterior-posterior and lateral roentgen graphic view of a 6 year old child's elbow before surgery.
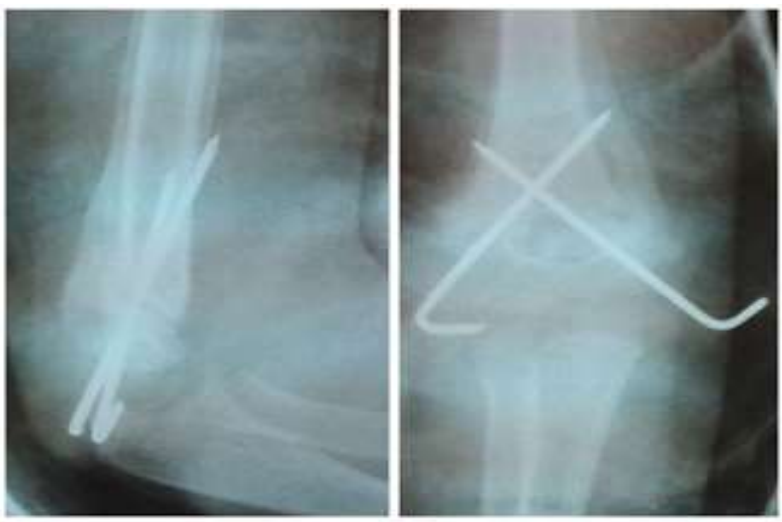

Figure 2: Anterior-posterior and lateral roentgen graphic view of a 6 year old child's elbow immediate post-surgery.
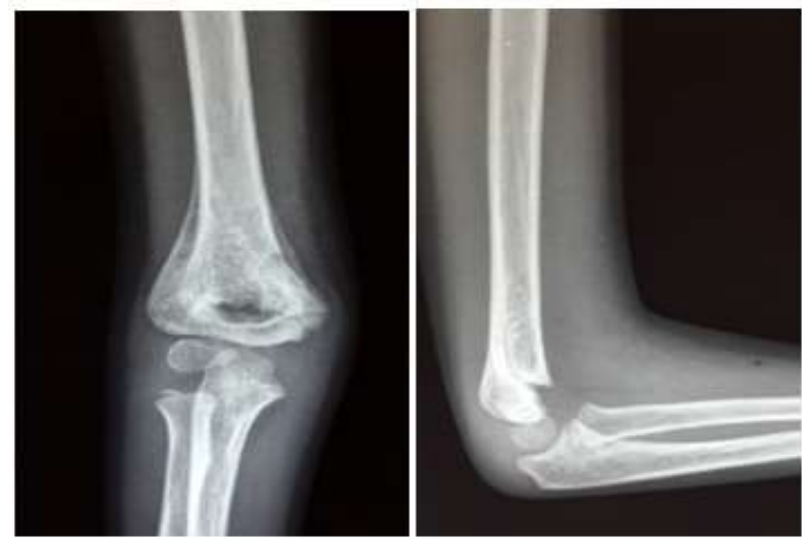

Figure 3: Anterior-posterior and lateral roentgen graphic view of a 6 year old child's elbow 24 weeks after the surgery. 


\section{RESULTS}

The average age of the patients were 6.4 years and maximum number of patients $18(72 \%)$ were in the 4-9 years age group. Out of the 25 patients, 17 (68\%) were males and $8(32 \%)$ were females. Most of the children $(52 \%)$ sustained injury falling while playing. All the fractures were closed, $16(64 \%)$ of which had posteromedial displacement and 9 (36\%) with posterolateral displacement.

Table 2: Mode of injury.

\begin{tabular}{|lll|}
\hline Nature of trauma & No. of patients & Percentage \\
\hline Fall from bicycle & 8 & 32 \\
\hline Fall from tree/steps & 4 & 16 \\
\hline Fall while playing & 13 & 52 \\
\hline
\end{tabular}

The average interval between injury and surgery was 1.2 days and the average stay in the hospital was 4.3 days.

Table 3: Loss of range of motion.

\begin{tabular}{|lll|}
\hline $\begin{array}{l}\text { Loss of range of } \\
\text { motion (degree) }\end{array}$ & No. of patients & Percentage \\
\hline $0-5$ & 16 & 64 \\
\hline $6-10$ & 6 & 24 \\
\hline $11-15$ & 2 & 8 \\
\hline$>15$ & 1 & 4 \\
\hline
\end{tabular}

Table 4: Carrying angle loss.

\begin{tabular}{|ll|l|}
\hline $\begin{array}{l}\text { Carrying angle } \\
\text { loss (degree) }\end{array}$ & No. of patients & Percentage \\
\hline $0-5$ & 18 & 72 \\
\hline $6-10$ & 6 & 24 \\
\hline $11-15$ & 0 & 0 \\
\hline$>15$ & 1 & 4 \\
\hline
\end{tabular}

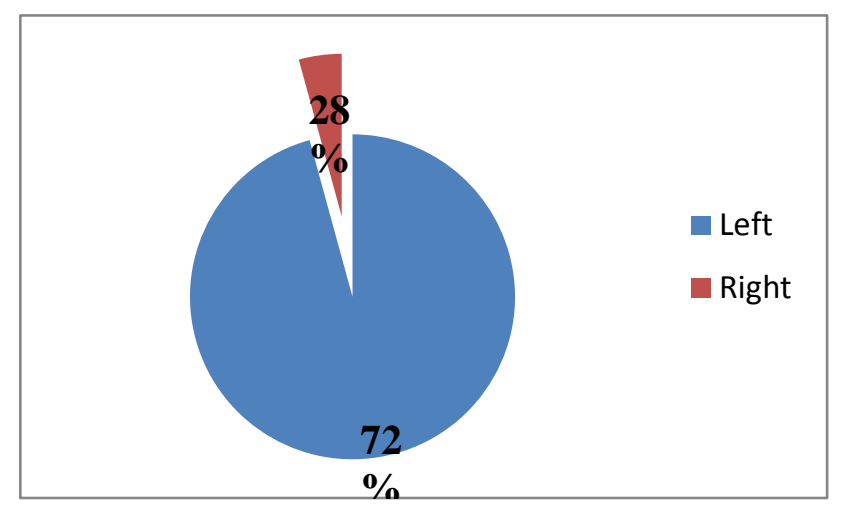

Figure 4: Side affected.

In our study, fractures of all the patients united by around 4 weeks time. Superficial pin tract infection was noticed in one patient who was treated with appropriate antibiotics.
The final results were evaluated using Modified Flynn's criteria. $16(64 \%)$ children were rated as excellent, 6 $(24 \%)$ as good, $2(8 \%)$ as fair and $1(4 \%)$ as poor result. Thus $96 \%$ of the patients had satisfactory results and only one had an unsatisfactory result.

\section{DISCUSSION}

The aims of the treatment of Type III supracondylar fractures of humerus in children are to achieve functionally and cosmetically satisfactory results and to avoid complications. The present study was aimed to discuss the age, sex and side incidence associated with these types of fractures and then anatomical and functional results and lastly complications associated with the treatment procedure.

Wilkins K.E reported the average age of the children with supracondylar fracture of humerus to be 6.7 years. $^{7}$ This was supported by the present study which showed $72 \%$ of patients were from 4-9 years age group and 6.4 years being the mean age.

The present study showed male predominance among patients with $68 \%$ as seen in earlier studies. This may be explained as boys are more active and more prone for falls. Flynn JC reported $66.7 \%$ fractures occurring on the left side. ${ }^{6}$ Present study also shows increased incidence of fractures occurring on the left side with $72 \%$.

Four major complications may be expected with closed reduction and percutaneous pinning with K-wires; these are superficial pin tract infection, iatrogenic ulnar nerve injury, K-wire migration and Cubitus varus deformity. Boyd had reported two cases of iatrogenic ulnar nerve injury. ${ }^{8}$ But the present study showed only a case of superficial pin tract infection which was treated with appropriate antibiotics.

\section{CONCLUSION}

In the present study, closed reduction and percutaneous pinning was performed in twenty five cases of type III supracondylar fracture of humerus in children out which $96 \%$ of the children had satisfactory results with superficial pin tract infection seen only in a single patient as complication. It is believed that the present technique is a safe and efficient method of fixation of type III supracondylar fracture of humerus in children.

Funding: No funding sources

Conflict of interest: None declared

Ethical approval: The study was approved by the institutional ethics committee

\section{REFERENCES}

1. Mostafavi HR. and Spero C. Crossed pin fixation of displaced supracondylar humerus fractures in children. Clin Orthop, 2000;376:56-61. 
2. Kasser JR, Beaty JH. Supracondylar fractures of the distal humerus. In Rockwood and Wilkins Fractures in children, 5th edition, Vol. 3, Philadelphia; Lippincott Williams and Wilkins; 2001:577-620.

3. Haddad RJ, Saer JK, Riordan DC. Percutaneous pinning of displaced supracondylar fractures of the elbow in children. Clin Orthop. 1970;71:112-7.

4. Pirone AM1, Graham HK, Krajbich JI. Management of displaced extension-type supracondylar fractures of the humerus in children. J Bone Joint Surg. 1988;70A;641-50

5. Canale TS ed. Fractures and dislocations in children. In Campbell's operative orthopaedics, $9^{\text {th }}$ edition, Vol. 3. New York; Mosby;1998:2407-2422.
6. Flynn JC, Mathews JG, Benoit RL. Blind pinning of displaced supracondylar fractures of the humerus in children. J Bone Joint Surg. 1974;56A:263-72.

7. Wilkins KE. The operative management of supracondylar fracture. Ortho Clin North Am. 1990;21(2);269-89.

8. Boyd DW, Aronson DD. Supracondylar fractures of the humerus: a prospective study of percutaneous pinning. J Pediatr Orthop. 1992;12:789-94.

Cite this article as: George PT, Kattoor MJ,

Edayalamuriyi SS. Treatment outcome of gartland type III supracondylar fracture humerus in children with closed reduction and percutaneous pinning. Int $\mathrm{J}$ Res Orthop 2016;2:95-8. 